\title{
A Coherent Through-the-Wall MIMO Phased Array Imaging Radar Based on Time-Duplexed Switching
}

\author{
Qingchao Chen ${ }^{\mathrm{a}}$, Kevin Chetty ${ }^{\mathrm{a}}$, Paul Brennan ${ }^{\mathrm{b}}$, Lai Bun Lok ${ }^{\mathrm{b}}$, Matthew Ritchie ${ }^{\mathrm{c}}$, Karl \\ Woodbridge ${ }^{b}$ \\ ${ }^{a}$ Dept. of Crime and Security Science, University College London, London, U.K.; \\ ${ }^{\mathrm{b}}$ Dept. of Electronics and Electrical, University College London, London, U.K.; \\ ${ }^{\mathrm{c}}$ Defense Science and Technology Lab, Salisbury, U.K.
}

\begin{abstract}
Through-the-Wall (TW) radar sensors are gaining increasing interest for security, surveillance and search \& rescue applications. Additionally, the integration of Multiple-Input, Multiple-Output (MIMO) techniques with phased array radar is allowing higher performance at lower cost. In this paper we present a 4-by-4 TW MIMO phased array imaging radar operating at $2.4 \mathrm{GHz}$ with $200 \mathrm{MHz}$ bandwidth. To achieve high imaging resolution in a cost-effective manner, the $4 \mathrm{Tx}$ and $4 \mathrm{Rx}$ elements are used to synthesize a uniform linear array (ULA) of 16 virtual elements. Furthermore, the transmitter is based on a single-channel 4-element time-multiplexed switched array. In transmission, the radar utilizes frequency modulated continuous wave (FMCW) waveforms that undergo de-ramping on receive to allow digitization at relatively low sampling rates, which then simplifies the imaging process. This architecture has been designed for the short-range TW scenarios envisaged, and permits sufficient time to switch between antenna elements. The paper first outlines the system characteristics before describing the key signal processing and imaging algorithms which are based on traditional Fast Fourier Transform (FFT) processing. These techniques are implemented in LabVIEW software. Finally, we report results from an experimental campaign that investigated the imaging capabilities of the system and demonstrated the detection of personnel targets. Moreover, we show that multiple targets within a room with greater than approximately 1 meter separation can be distinguished from one another.
\end{abstract}

Keywords: Multiple Input Multiple Output Radar, Phase Array, Radar Imaging, Through Wall, Time-Duplexed radar.

\section{INTRODUCTION}

The ability to detect the presence of individuals behind visually obscuring structures is of significant benefit to police, security and emergency agencies in many situations. Significant amount of research has attempted to apply radar detection and imaging techniques to scenarios that consist of an obscuring wall barrier. Through-Wall (TW) radars have been employed in short range detection applications which are generally used to detect the motion, location and signs of life indicators of human targets inside buildings.

To detect a moving target, Continuous Wave (CW) radars can be utilized to detect only the Doppler information related to target's movement. CW radar can be regarded as only a motion detector without providing any range information. However, the micro-Doppler signature can still be utilized to differentiate the target's motions with the help of recognition algorithms. Single channel FMCW radar has been used to detect the range of the target behind the wall but not any angle information, which can only provide the 1-D information. Therefore, the detection capability is limited by the beam-width of the antenna. To detect 2-D or even 3-D information of the target behind the wall, Peabody et al. [1] designed a UWB FMCW MIMO radar ranging from $2 \mathrm{GHz}$ to $4 \mathrm{GHz}$ and a 8 transmit 13 receive phased antenna array to generate the radar image behind the wall. The chirp generation of this radar is built via a ramp generator and the voltage control oscillator and the transmit peak power is $2 \mathrm{~W}$. In addition, the range gating method is implemented as a band pass filter. Dilsavor et al. [2] built a VNA-based synthetic aperture radar by moving the single transmit and single receive antenna and post process these data by applying different weights corresponding to their antenna positions. However, this VNA-based radar lacks the ability to detect moving targets behind the wall as they need to move their one channel Tx and Rx antenna to form the synthetic aperture. Another combination of the SAR processing and phased array MIMO

Radar Sensor Technology XXI, edited by Kenneth I. Ranney, Armin Doerry, Proc. of SPIE Vol.

10188, $1018809 \cdot$ @ C 2017 SPIE · CCC code: 0277-786X/17/\$18 · doi: 10.1117/12.2262462 
radar is to mount the antenna arrays and radar on the truck [3]. The truck moves in the azimuth direction and the array is in the elevation direction to obtain the 3-D imaging capabilities.

The most similar work to us in the radar design is Huang et al. [4], who designed a time-duplexed phased array MIMO system for maritime navigation. However, their system was not designed to operate with parameters commensurate for through-wall stand-off detection scenarios. The array design between the system is also different. The work from Peabody et al. is also similar to our work in terms of employing time-duplexed MIMO techniques however the radar design is different: we use a DDS to generate the $200 \mathrm{MHz}$ linear chirp whilst they utilized a Voltage Controlled Oscillator (VCO) to generate the Ultra-Wide-Band (UWB) chirps of $2 \mathrm{GHz}$ bandwidth.

In this paper, we present a TW FMCW Phased-Array MIMO radar centered at $2.4 \mathrm{GHz}$ with $200 \mathrm{MHz}$ bandwidth and the conventional patch antenna array. To build a low-cost MIMO radar, we make use of switching technique to form multiple virtual array elements [5]. Next, we apply FFT based array processing to the MIMO data. Moreover, we demonstrate the imaging capability of the system using targets moving behind a wall. To achieve this result, we also employ a moving target indication (MTI) filter to mitigate the effects of the wall. In section 2, we describe the signal model of the switched phased array MIMO radar and signal processing methods to obtain the radar images. In section 3, the design and implementation of the radar system and the antenna array are explained. Next in section 4, the imaging results from an experimental trial are illustrated, including single and multiple moving targets. Finally in section 5, we conclude the paper by summarizing the main advancements from the research.

\section{SIGNAL MODEL AND PROCESSING METHOD}

\subsection{Model of Phased Array Radar}

The through-the-wall (TW) MIMO phased array radar described in this paper consists of a four element transmitter (TX) array and a four element receiver (RX) array. To minimize direct signal interference (DSI) the arrays are separated by $1 \mathrm{~m}$, and offset by a height of $1.4 \mathrm{~m}$. Both the TX and RX arrays have equal element spacings of $2.4 \lambda$ and $0.6 \lambda$ respectively. This configuration synthesizes a 16 element virtual uniform linear array (ULA) with an equal element spacing of $0.3 \lambda$, and was chosen to avoid grating lobes by a large margin in the MIMO array processing. Figure 1 illustrates the real and virtual synthesized elements in the antenna arrays.

Consider $N_{t}$ elements in the TX array and $N_{r}$ elements in the RX array where $t=4$ and $r=4$. Their physical coordinates are denoted as $x_{i}^{T X}, i=1,2,3,4$, and the coordinates of the $\mathrm{RX}$ are denoted as $x_{j}^{R X}, j=1,2,3,4$. Assuming that the far field condition is met, then the round trip distance $P_{i j}$ between the $i^{\text {th }}$ element in TX array to the $j^{\text {th }}$ element in RX array via a point scatterer at point $\boldsymbol{p}$ is approximated as twice of the distance between the virtual element $x_{i j}=\left(x_{i}^{T X}+x_{j}^{R X}\right) / 2$ and the point scatter $\boldsymbol{p}[6,7]$ :

$$
P_{i j}=\left\|p-x_{i}^{T X}\right\|_{2}+\left\|p-x_{j}^{R X}\right\|_{2}=2 \times\left\|p-x_{i j}\right\|_{2}
$$

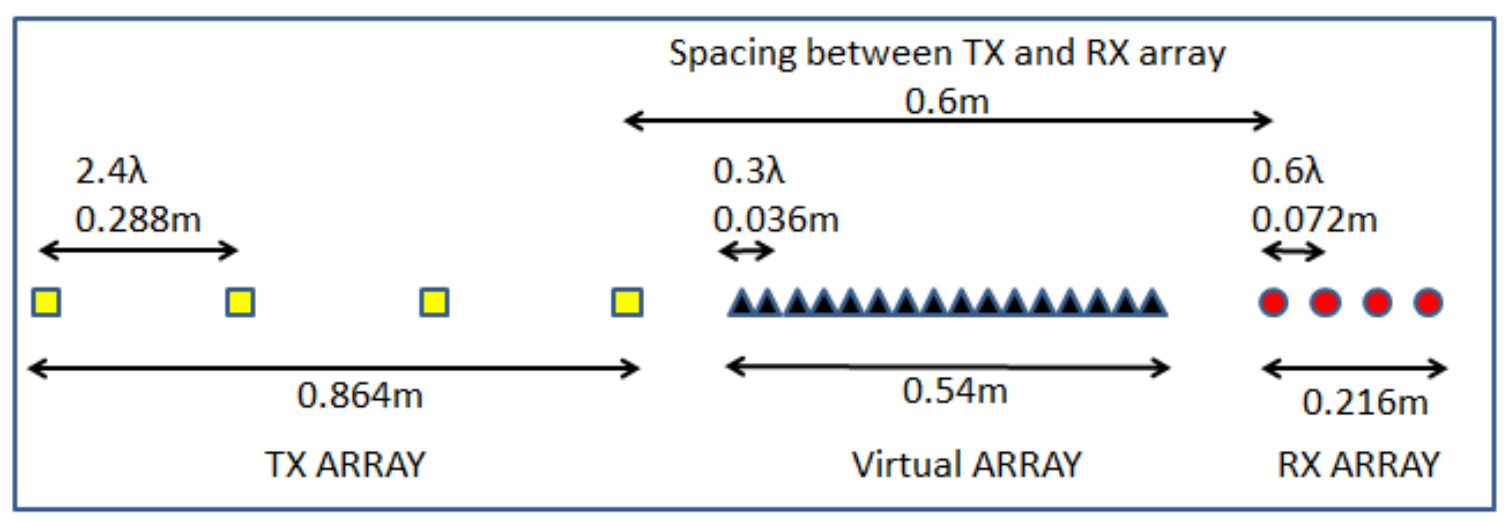

Figure 1. Placement of the Phased array, including the TX Array, RX Array and the Virtual Array. 


\subsection{Signal Model of the MIMO FMCW Radar}

The signal transmitted by the MIMO radar is a linear frequency continuous wave $s(t)$, with the carrier frequency $f_{c}$, bandwidth $B$ and sweep time $T$. For the nth chirp the signal $s_{n}(t)$ can be represented as the following:

$$
s_{n}(t)=A_{o} \exp \left(j 2 \pi f_{c}(t-(n-1) T)\right) \times \exp \left(j \pi \mu(t-(n-1) T)^{2}\right) \times u(t-(n-1) T),
$$

where $A_{0}$ denotes the amplitude of the transmitted signal, $\mu=B / T$ is the chirp rate and $u(t)$ is a rectangular function defined as:

$$
u(t)=\left\{\begin{array}{c}
1,0<t<T \\
0, \text { others }
\end{array}\right\} .
$$

Suppose the far field condition is met, the return delay between virtual element $x_{i j}$ and a point scatterer at the range $R$ and the angle $\theta$ (corresponding to the boresight) can be represented as:

$$
\tau_{i j}=\frac{2 R}{c}+\frac{2 x_{i j} \sin \theta}{c}
$$

where $\mathrm{c}$ is the speed of the light. Then the $n^{\text {th }}$ received chirp signal can be expressed as:

$$
r_{i j, n}(t)=A_{0} K_{r} s_{n}\left(t-\tau_{i j}\right)+z(t),
$$

where the amplitude of the received signal is $A$, the reflection coefficient of the target is $K_{r}$ and $z(t)$ is the noise signal. Finally, after de-ramp processing and low pass filtering, the beat signal can be expressed as [8]:

$$
b(t)=s_{n}(t) \times r_{i j, n}{ }^{*}(t)=K_{r} A_{0}^{2} \exp \left(j 2 \pi f_{c} \tau_{i j}\right) \times \exp \left(-j \pi \mu \tau_{i j}^{2}\right) \times \exp \left(j 2 \pi \mu \tau_{i j}(t-(n-1) T)\right) .
$$

From Eq.(6), it can be seen that the beat signal consists of three terms that incorporate both the range and angle information of the target. Firstly, the $\exp \left(j 2 \pi \mu \tau_{i j} t\right)$ term represents the frequency of the beat signal and indicates the range of the target as the follows

$$
f_{i j}(t) \approx \exp \left(\frac{j 4 \pi \mu R}{c} t\right) .
$$

It is worth noting that, due to the far field assumption, the return delay measured by all virtual elements $\tau_{i j}$ are approximately equal to $2 R / c$. Additionally, the range resolution is proportional to the inverse of the chirp duration $T$.

Secondly, the second and third phase terms $\exp \left(j 2 \pi f_{c} \tau_{i j}\right)+\exp \left(-j \pi \mu \tau_{i j}{ }^{2}\right)$ in Eq.(6) indicates the bearing of the target. In the application of TW imaging, within the detectable range, the square phase term $\exp \left(-j \pi \mu \tau_{i j}{ }^{2}\right)$ can be approximated as the constant due to the relatively small distance difference among the array elements and the square function as well. Therefore, the phase term of the de-ramped signal can be expressed as:

$$
\varphi=2 \pi f_{c}\left(\frac{2 R}{c}+\frac{2 x_{i j} \sin \theta}{c}\right)-\pi \mu \tau_{i j}^{2}=\frac{4 \pi f_{c} x_{i j} \sin \theta}{c}+C=\frac{4 \pi x_{i j} \sin \theta}{\lambda}+C,
$$

where $C$ is the constant. The $4 \pi / \lambda$ then validates the two-way path delay and suggests that designing the TX and RX arrays such that the inter-element spacing in the virtual array is $0.3 \lambda$, would generate the minimum grating lobes around +-74 degree with the scanning angle from -45 to 45 degree. However, in the practical TW applications, as the patch antenna would have the $3 \mathrm{~dB}$ beam-width around 100degree, these grating lobes would be suppressed.

\subsection{Signal Processing Method for 2-D Imaging}

The frequency spectrum $U(f)$ and the range information $U(R)$ in Eq.(6) and Eq.(7), can be estimated by performing the Fast Fourier Transform (FFT) or any other matching pursuit algorithms, such as MUSIC on the beat signal $b(t)$. Moreover, the steering vector of corresponding to the $i^{\text {th }}$ element in TX and $j^{\text {th }}$ element in RX, denoted as $a_{i j}$ can be denoted as: 


$$
a_{i j}(\theta)=\exp \left(-j 4 \pi \frac{x_{i j} \sin \theta}{\lambda}\right) .
$$

In this way, the azimuth profile, or the angular profile $P(\theta)$ can be estimated as the following:

$$
\mathrm{P}(\theta)=\sum_{\mathrm{i}=1}^{\mathrm{N} t} \sum_{j=1}^{N r} a_{i j}(\theta) U(f) .
$$

It is worth noting that FFT, MUSIC and any method related to the matching pursuit can be utilized to obtain the range and the azimuth profiles.

As illustrated in section 2.2, the range resolution is related to the frequency resolution, therefore, the range resolution is limited by the bandwidth of the chirp, as the following equation shows:

$$
\Delta R=\frac{c}{2 \mu} \times \frac{1}{T}=\frac{c}{2 B} .
$$

The angular resolution is then dependent on both the aperture of the MIMO virtual array and the given angle $\theta$ according to the following equation:

$$
\Delta \theta=\frac{\lambda}{2 A_{e}}=\frac{\lambda}{2 d(N t N r-1) \cos \theta},
$$

where $d$ is the inter-element spacing of the virtual array and $A_{e}$ is the virtual aperture of the antenna array. Furthermore, the azimuth resolution at the range $R$ and the given angle $\theta$ can be estimated as:

$$
\Delta R_{\text {azimuth }} \approx R \times \Delta \theta=\frac{\lambda R}{2 d(N t N r-1) \cos \theta} .
$$

\subsection{Switching Technique for Expanding the MIMO Channels}

The radar consists of a direct digital synthesizer (DDS) which is connected directly to a four-way switch each of which feeds a radiating element $N t$ in the TX array. This enables MIMO signal transmissions through time-duplexing. Here, the scanning time and chirp duration are limited by the motion of the target of interest. Firstly, in terms of the switching schemes, the target is restricted to a movement of less than a quarter of the wavelength whilst the system is scanning. That is to say, the maximum velocity of the target $v$ is defined as:

$$
v \times T \times(N t-1)<\lambda / 4 .
$$

In this way, the upper bound of the chirp sweep time $T_{u l}$ is limited by:

$$
T_{u 1}<\frac{\lambda}{4 v_{\max }(N t-1)} .
$$

The sweep period is also limited by the acceleration of the target to ensure coherency. It is defined that the change of the radian frequency $f_{d}$ is over the chirp period should be less than the frequency resolution, 1/T. The Doppler frequency $f_{d}$ of the target is:

$$
f_{d}=\frac{2 v}{\lambda},
$$

Thus the change of the Doppler frequency within the chirp period can be expressed as:

$$
\Delta f_{d}=\frac{2}{\lambda} \frac{d v}{d t} T=\frac{2 a}{\lambda} T<\frac{1}{T} .
$$

This gives the second upper bound of the chirp period, $T_{u 2}$ as the following: 


$$
T_{u 2}<\sqrt[2]{\frac{\lambda}{2 a_{\max }}}
$$

\section{HARDWARE IMPLEMENTATION}

In this section, firstly, the phased array antenna is illustrated and secondly, the system architecture of the proposed switched MIMO FMCW radar is shown. In general, the phased array radar consists of 4-element TX antenna array and a 4-element RX antenna array. The radar is centered at $2.5 \mathrm{GHz}$ and the bandwidth is $200 \mathrm{MHz}$, with the chirp time of $1 \mathrm{~ms}$.

\subsection{Design of the Phased Array Antenna}

A patch antenna was chosen due to its ease of fabrication and with the acceptable 90 degree azimuth beam-width. However, due to the $3 \%$ bandwidth limitation of the normal patch antenna, we utilized the corrugation technique along the width of the patch antenna to increase the bandwidth. The following Figure 2 shows the S11 reflection coefficient plot with $-7.18 \mathrm{~dB} \mathrm{~S} 11$ between $2.4 \mathrm{GHz}$ and $2.6 \mathrm{GHz}$, therefore the patch antenna designed is suitable for the requirement of the radar.

As the signal transmitted is in the range of $2.4 \mathrm{GHz}$ and $2.6 \mathrm{GHz}$, the element spacing in the TX is set as the 2.4 times of the wavelength at the frequency of $2.5 \mathrm{GHz}$, which is 0.288 meter and the element spacing in the RX is set to 0.6 of the wavelength, which is 0.072 meter.

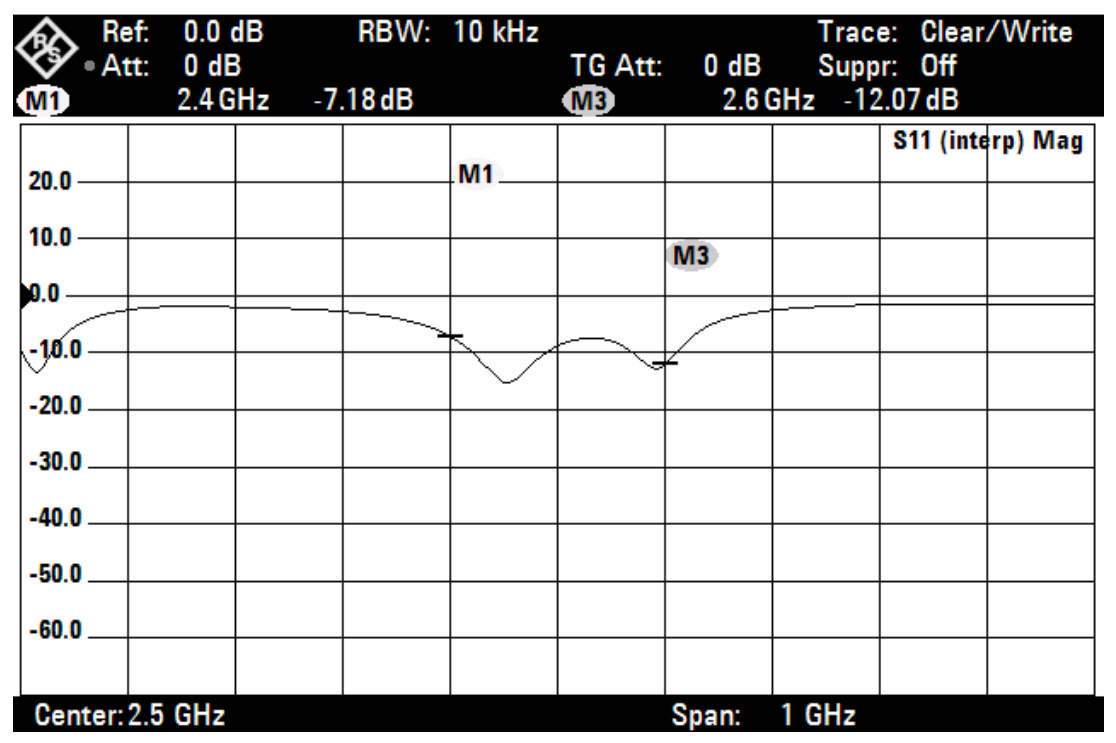

Figure 2. S11 measurement from the Vector Network Analyzer of the single patch antenna; measurement obtained here are -7.18dB at $2.4 \mathrm{GHz}$ and $-12.07 \mathrm{~dB}$ at $2.6 \mathrm{GHz}$. The bandwidth is approximately $210 \mathrm{MHz}$, with the $-8 \mathrm{~dB} \mathrm{~S} 11$ measurement.

\subsection{System Architecture of the MIMO FMCW Radar}

The system architecture of the MIMO FMCW radar is illustrated in Figure 3. First, the VCO and PLL are utilized to generate a Continuous Wave waveform at $2 \mathrm{GHz}$ and this signal will be fed to the DDS for generating the linear chirp signal, with $1.024 \mathrm{~ms}$ chirp period and $200 \mathrm{MHz}$ bandwidth, sweeping from $2.4 \mathrm{GHz}$ to $2.6 \mathrm{GHz}$. After this, the bandpass filter and the amplifier are utilized to filter out undesired signals and amplify the desired signal. A splitter is then used to separate the signal into two channels, one is used for the reference signal to the mixer, prepared for the de-ramp processing, while the other is connected to the four-way switch to expand the switching array.

The return signal is collected from RX array and fed into the bandpass filter with the amplifier, and finally to the mixer. After that, the active filter is utilized to clean and amplify the de-ramped signal related to the range information. Finally the de-ramped signal is digitized by the ADC and passed to the PC by USB cable for the imaging processing. 


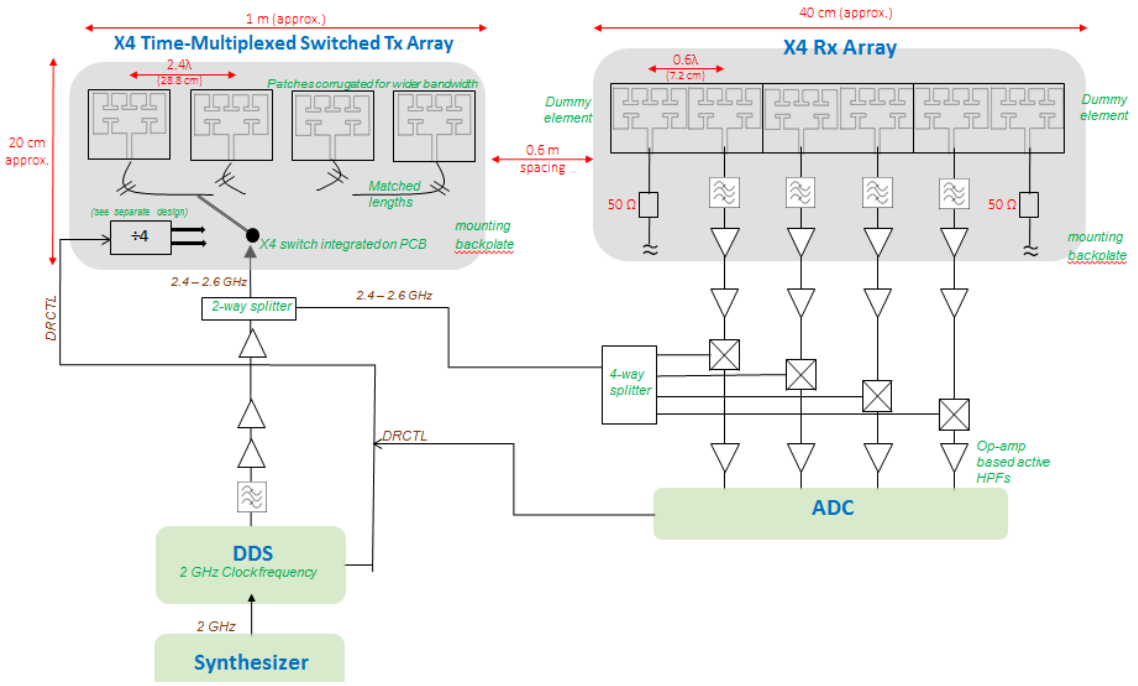

Figure 3. System architecture of the proposed MIMO Radar

\section{EXPERIMENTAL RESULTS AND ANALYSIS}

In this section, we present the experimental results of both single input single output (SISO) and MIMO set up in the following scenario. In section 4.1, the experiment set-up and in section 4.2, the results of both the SISO and MIMO processing are shown and analyzed.

\subsection{Experiment Set-Up}

The experiment is set up in the cluster room and the corridor in University College London. As shown in Figure 4, the TX and RX arrays are $1.5 \mathrm{~m}$ away from the $10 \mathrm{~cm}$ depth plastic wall perpendicularly. The TX and RX arrays are tha $0.4 \mathrm{~m}$ away from each other. In addition, there are five test positions ranging from P1 to P5 in the corridor in case of the clutters in the cluster room. It is worth noting that the corridor is approximately $2.2 \mathrm{~m}$ long.

For each position in the experiment, a target is walking in the corridor as shown in the double arrows, from one end of the wall to the other end perpendicularly to the wall as well.

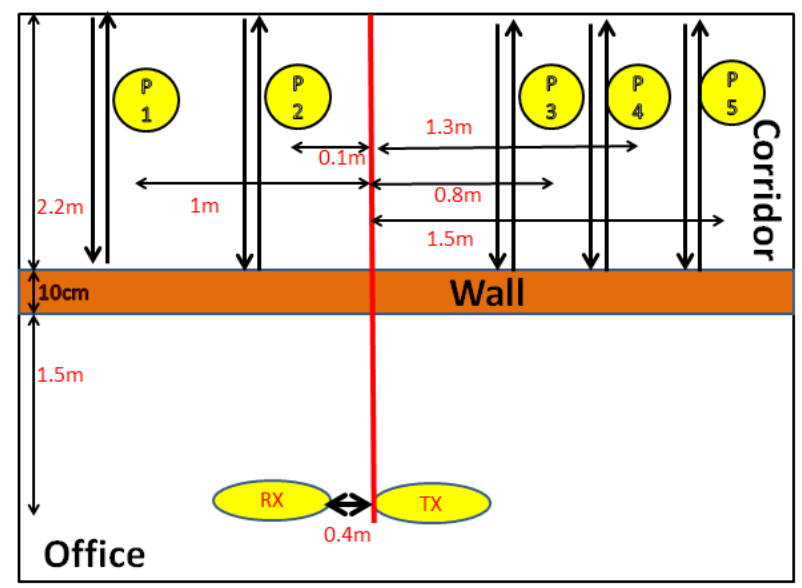

Figure 4. Experiment Set Up; The radar is placed $1.5 \mathrm{~m}$ away from the $10 \mathrm{~cm}$ depth wall and the Tx and Rx part of the radar is $0.4 \mathrm{~m}$. The two arrows next to the position indicator represent the walking route of the target at 5 positions. The walking route of the target at each position is from one end of the wall to the other end. The detailed geometry can be shown in this figure. 


\subsection{Results}

First we present the range-time results without using the MTI filters as shown in Figure 5 (a) in the Single Input Single Output (SISO) mode. It is worth noting that this result is processed by only one TX channel and RX channel from the same experiment data as shown in the MIMO results. In Figure 5 (a), we can clearly see three static lines in the rangetime results, the first of which is the DSI near $0 \mathrm{~m}$. The strongest line at approximately $1.5 \mathrm{~m}$ is attributed to the first wall between the corridor and the office. There is a weaker static line around $3.85 \mathrm{~m}$ which we regarded as the response from the second wall.

To detect the moving target behind the wall, we applied a Finite Impulse Response (FIR) digital filter on the slow time de-ramp signal and then performed the FFT based array processing. The relevant moving target result for Figure 5 (a) is shown in Figure 5 (b) and (c), where Figure 5 (b) clearly shows the walking pattern among the 5 different positions. The final part of the MTI range-time result between 35 and 45 seconds indicates the result of a human target walking across the corridor to get back to the office. Figure 5 (c) is the zoomed-in figure showing the detailed signatures from the positions $\mathrm{P} 2$ and $\mathrm{P} 3$.

It could be shown that in Figure 5 (b) that the power of the range-time results in P2 and P3 are stronger than other positions and P1 exhibits the lowest power. We hypothesize that this occurs because P2 and P3 are closer to the boresight of the antenna arrays. In addition, Figure 5 (c) displays some walking pattern signatures from 10s to $15 \mathrm{~s}$ in the range of $4 \mathrm{~m}$ to $6 \mathrm{~m}$ which is out of the physical limits of the corridor. We explain this in terms of the double bounce effect: as $\mathrm{P} 3$ is close to the boresight of the antenna array, the double bounced signal might exhibit higher powers.

Next, the MIMO 2-D images of the 5 positions are shown in Figure 6 (a) to (e) respectively. Here, the plots are normalized and shown with $-5 \mathrm{~dB}$ to $0 \mathrm{~dB}$ dynamic range. It can be seen from the 5 figures that different positions exhibit different angle estimation results. It is worth noting that the signal clutter ratio in Figure 6 (a) is lower as there is less power reflected from the P1. This corresponds to the SISO result shown in Figure 5 (b). Finally, as shown in Figure 7 we present the MIMO 2-D image of two targets walking in the opposite direction. In this scenario, the right target exhibits less power than the left target as it's further away from the radar.

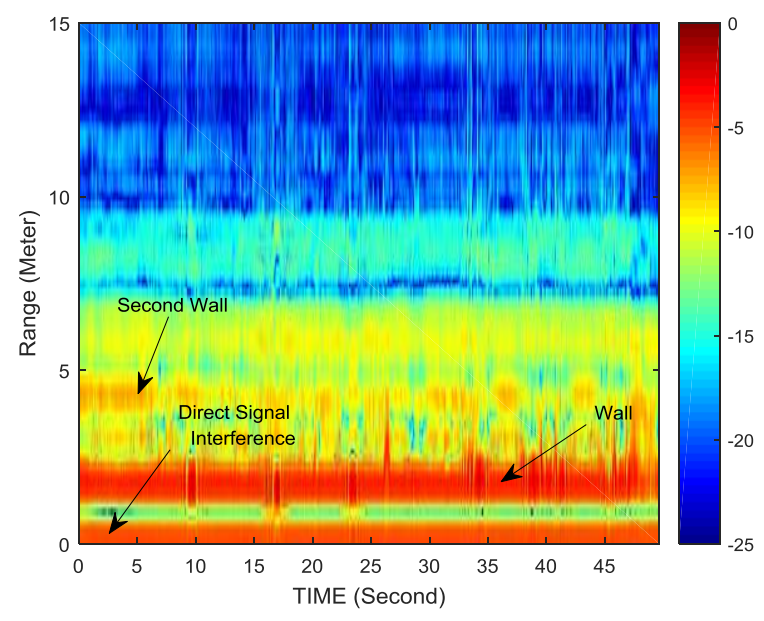

(a)

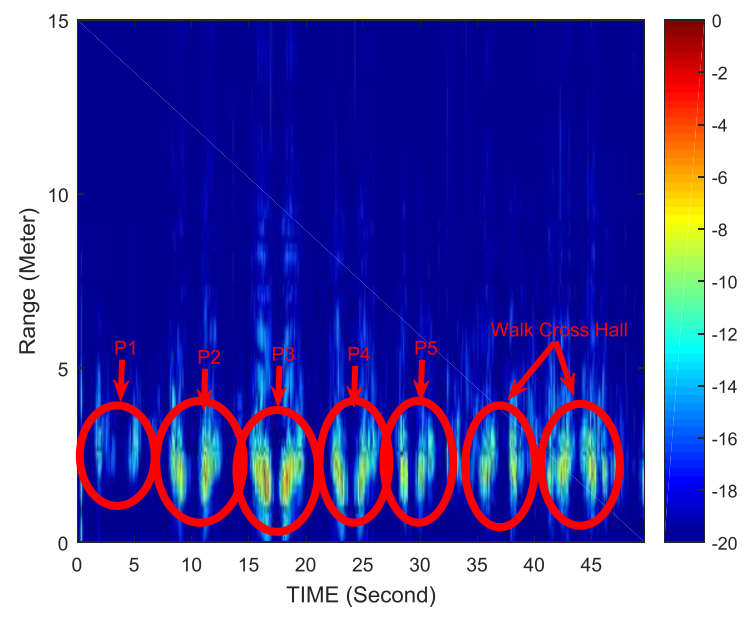

(b) 


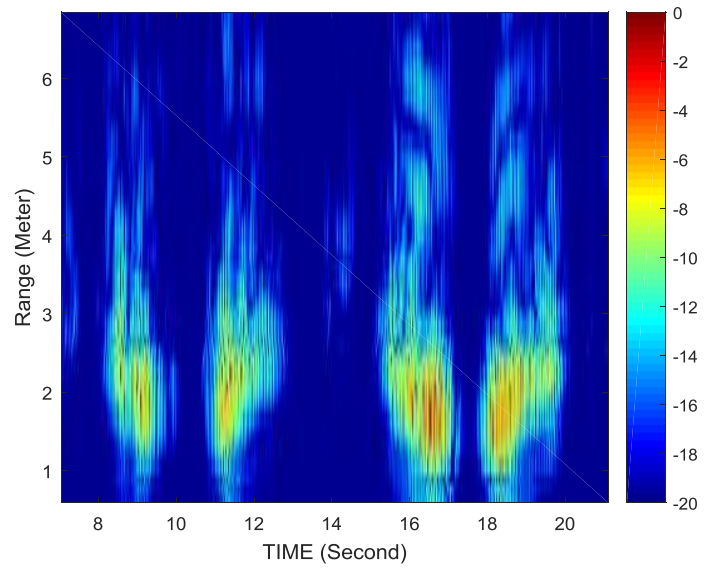

(c)

Figure 5. Single Input and Single Output Range-Time Plot of scenario 1: (a) result without wall mitigation technique (b) result with moving target indicator filter applied (c) Zoomed-in Results of figure 5 (b), for targets walking in P2 and P3.
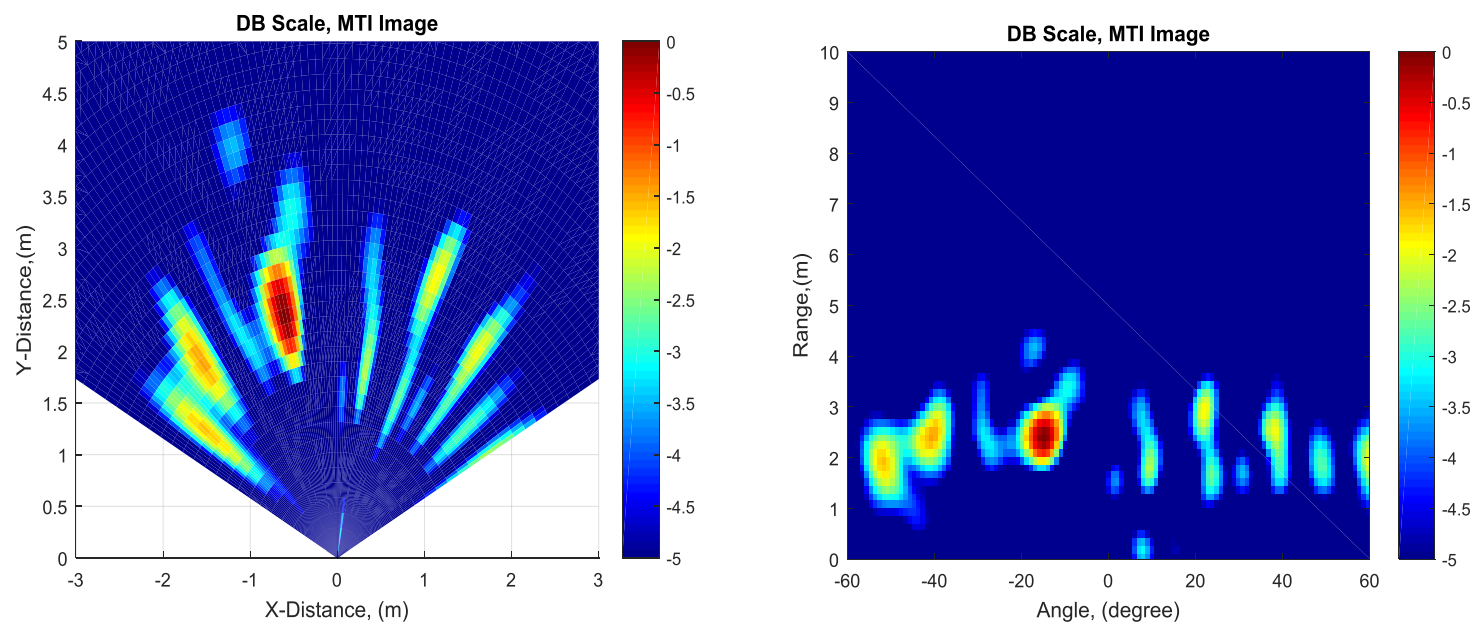

(a)
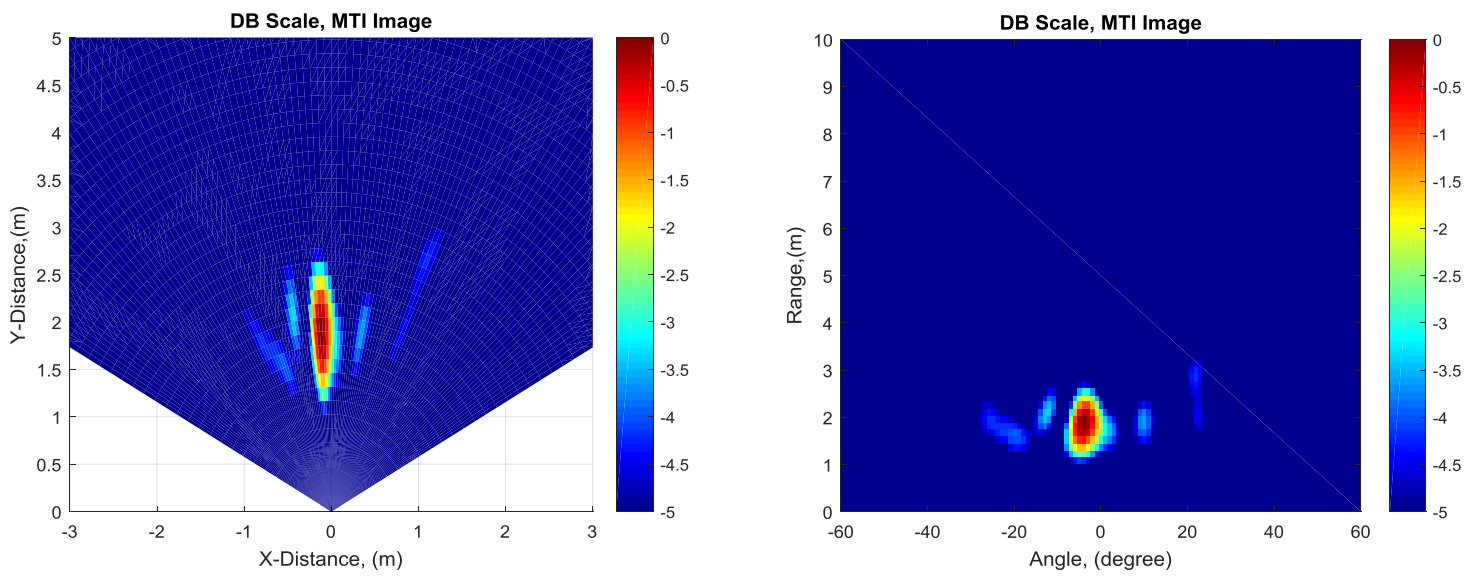

(b) 

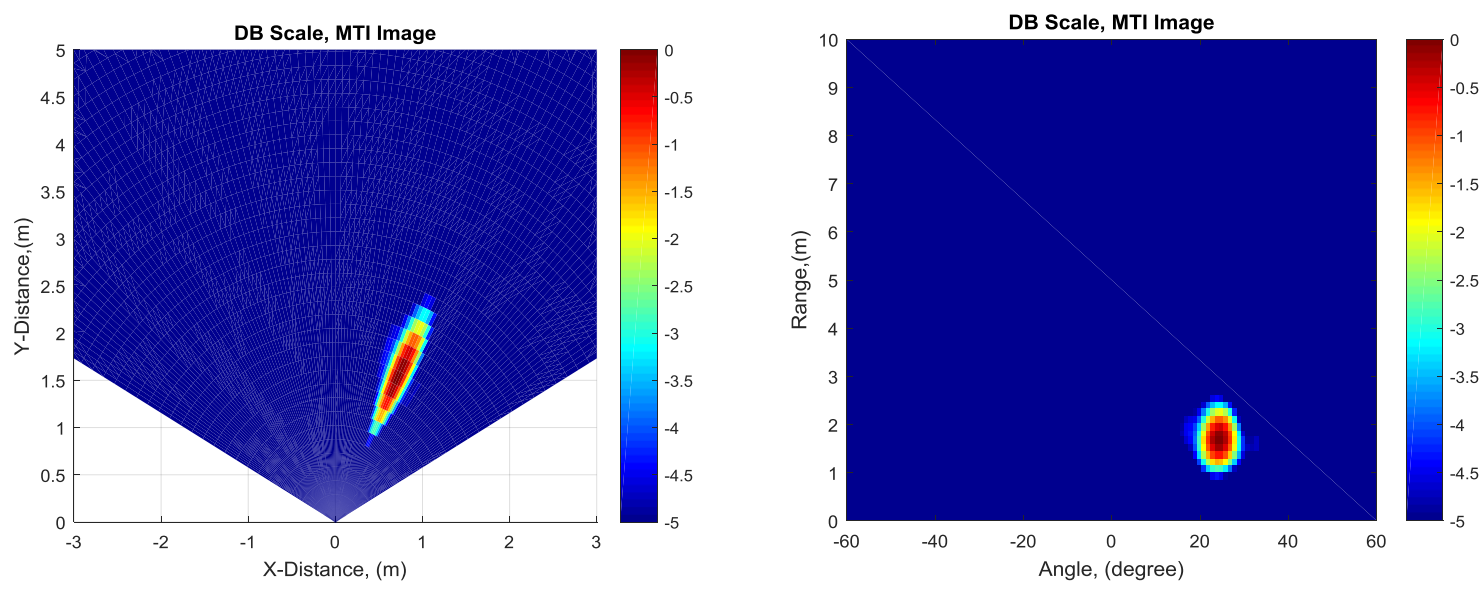

(c)
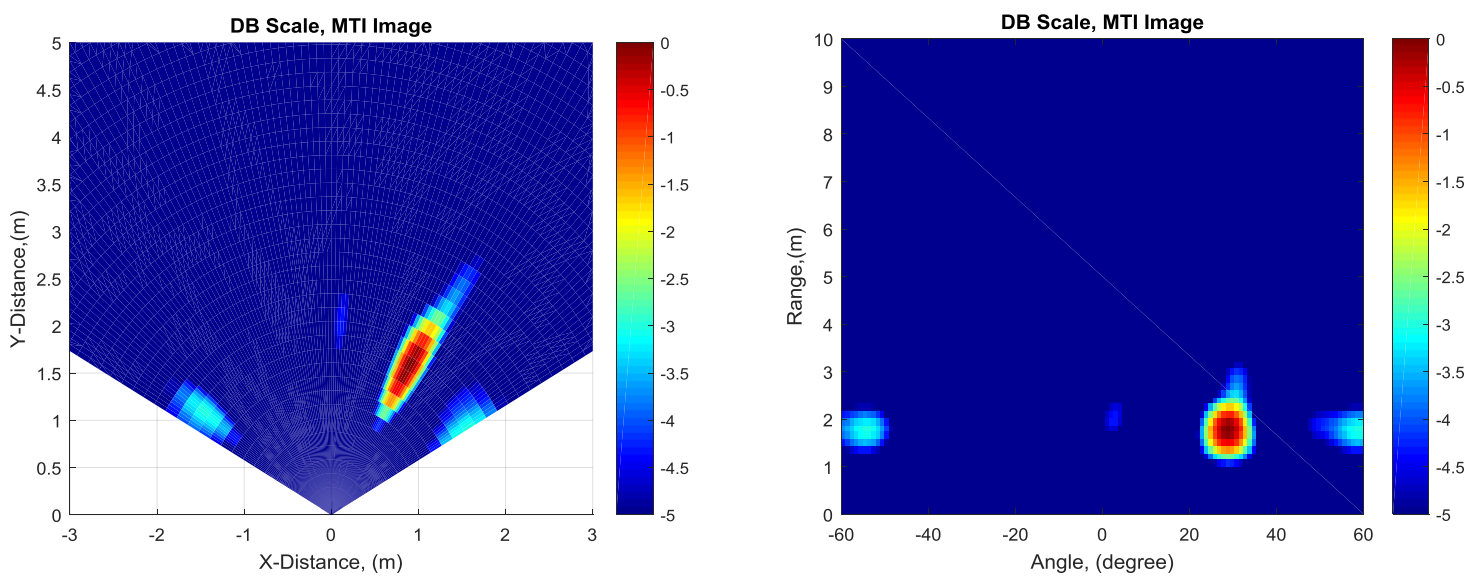

(d)
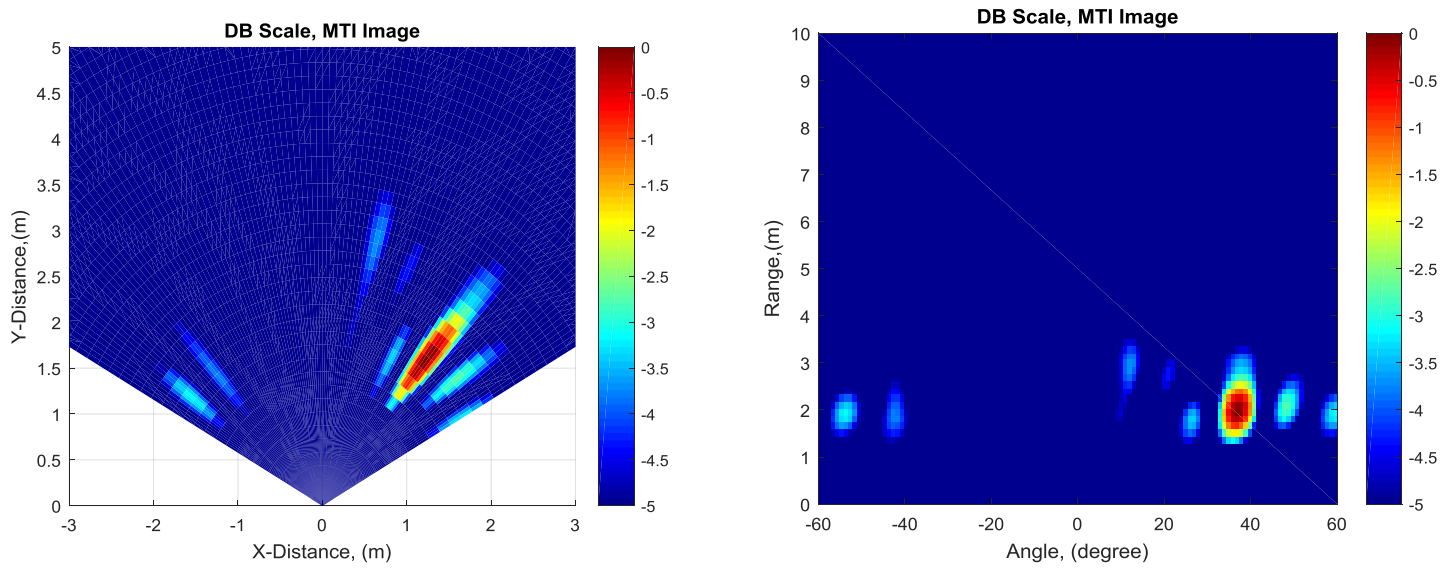

(e)

Figure 6. Through-Wall Imaging Results Among Five Different Positions in the hall: (a) P1, (b) P2, (c) P3, (d) P4, (e) P5. 

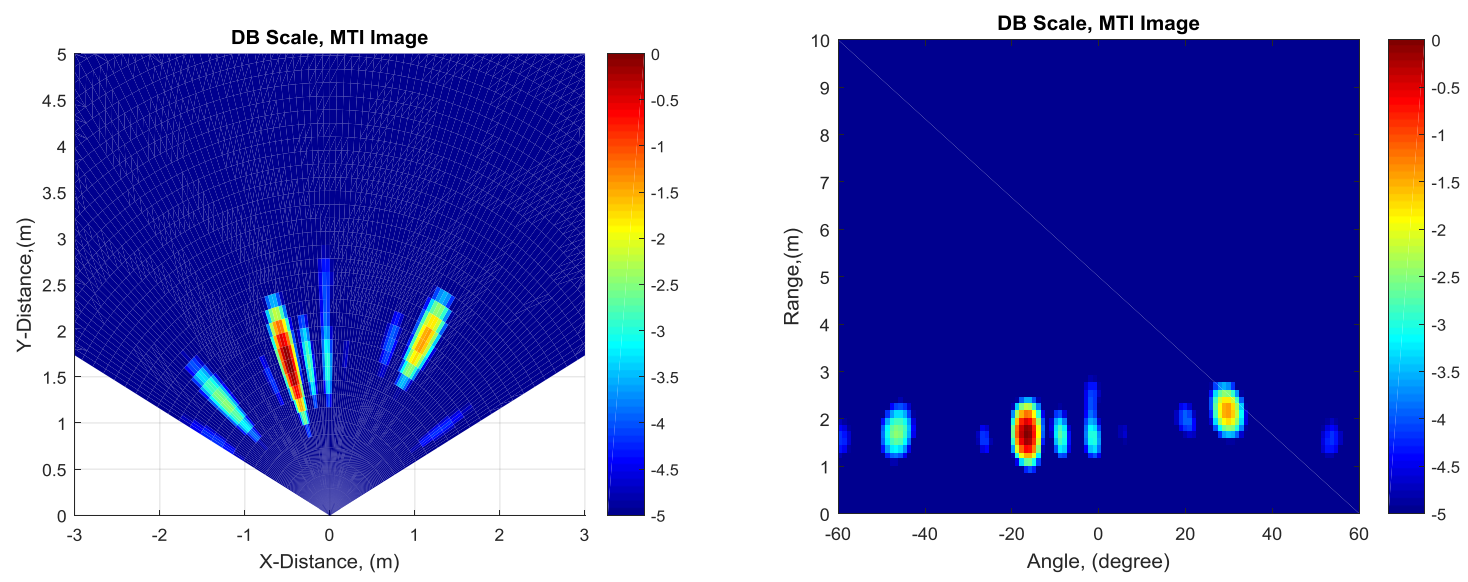

(a)
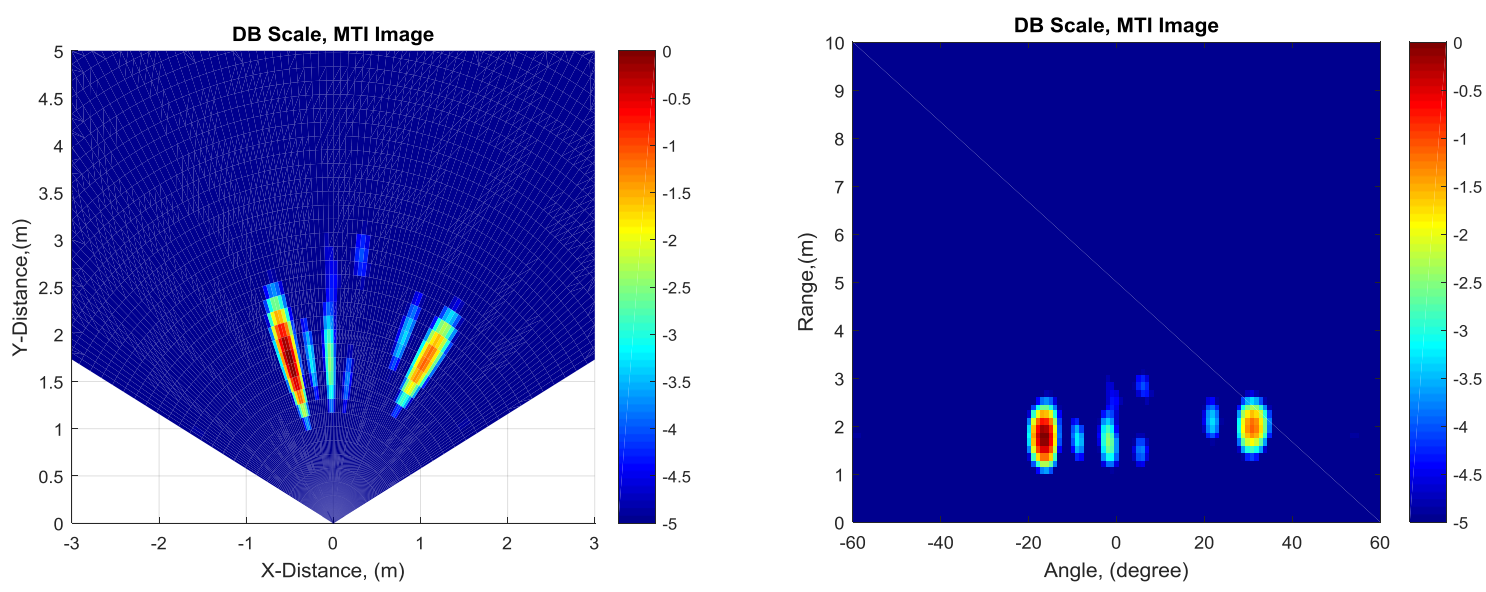

(b)

Figure 7. MIMO Imaging Results of Two Moving Targets with the right target approaching to the wall and the left target leaving away from the wall.

\section{CONCLUSION}

In this paper, we demonstrated a time-duplexed MIMO phased array FMCW radar design and the imaging results in a through-wall scenario. We fabricated the Tx and Rx array using the patch antenna arrays and the FMCW radar is centered at $2.5 \mathrm{GHz}$ and with bandwidth of $200 \mathrm{MHz}$. It successfully demonstrated the imaging capability of the moving target in a through-wall scenario. Future work would focus on investigating imaging capabilities among various wall types and through-wall imaging capabilities in the stand-off distances.

\section{REFERENCES}

[1] J. E. Peabody, G. L. Charvat, J. Goodwin, and M. Tobias, "Through-wall imaging radar," Lincoln Lab. J, vol. 19, pp. $62-72,2012$.

[2] R. Dilsavor, W. Ailes, P. Rush, F. Ahmad, W. Keichel, G. Titi, et al., "Experiments on wideband through-the-wall radar imaging," in Defense and Security, 2005, pp. 196-209. 
[3] G. B. Barrie, "Through-wall synthetic aperture radar (TWSAR) 3D imaging: algorithm design," DTIC Document2004.

[4] Y. Huang, P. V. Brennan, D. Patrick, I. Weller, P. Roberts, and K. Hughes, "FMCW based MIMO imaging radar for maritime navigation," Progress In Electromagnetics Research, vol. 115, pp. 327-342, 2011.

[5] J. Li, P. Stoica, and X. Zheng, "Signal synthesis and receiver design for MIMO radar imaging," IEEE Transactions on Signal Processing, vol. 56, pp. 3959-3968, 2008.

[6] Y. Qu, G. Liao, S.-Q. Zhu, X.-Y. Liu, and H. Jiang, "Performance analysis of beamforming for MIMO radar," Progress In Electromagnetics Research, vol. 84, pp. 123-134, 2008.

[7] J. H. Ender and J. Klare, "System architectures and algorithms for radar imaging by MIMO-SAR," in Radar Conference, 2009 IEEE, 2009, pp. 1-6.

[8] P. Lacomme, Air and spaceborne radar systems: An introduction: William Andrew, 2001. 